\title{
NOTE ON NILPOTENT DERIVATIONS
}

\author{
P. H. LEE AND T. K. LEE
}

\begin{abstract}
Let $R$ be a prime ring with center $Z$. Suppose that $d$ is a derivation on $R$ such that $d^{n}(x) \in Z$ for all $x$, where $n$ is a fixed integer. It is shown that either $d^{n}(x)=0$ for all $x \in R$ or $R$ is a commutative integral domain. Moreover, the same conclusion holds even if we assume that $d^{n}(x) \in Z$ merely for all $x \in I$, where $I$ is a nonzero ideal of $R$.
\end{abstract}

Let $R$ be a prime ring with center $Z$ and a derivation $d$. Suppose that $d(x) \in Z$ for all $x \in R$, then one can easily show that either $d=0$ or $R$ is commutative [3, Proof, Theorem 2]. If $d^{2}(x) \in Z$ for all $x \in R$, we have the same conclusion when $R$ is not of characteristic 2 [3, Theorem 3]. On the other hand, in case char $R=2$ there exist nonzero derivations $d$ on $R$ such that $d^{2}=0$ provided $R$ is not a domain. One might naturally ask what we can conclude in general if $d^{n}(x) \in Z$ for all $x \in R$, where $n$ is some fixed integer. Certainly, this condition is fulfilled when $d$ is nilpotent of nilpotency $n$ or when $R$ is a commutative ring. We prove here that these are indeed the only two possibilities where $d^{n}(R) \subseteq Z$ can happen.

THEOREM 1. Let $R$ be a prime ring with center $Z$ and $d$ a derivation on $R$ such that $d^{n}(R) \subseteq Z$ for some natural number $n$. Then either $d^{n}=0$ or $R$ is commutative.

Proof. First note that if $Z=0$ there is nothing to prove. So we assume that $Z \neq 0$ and proceed to prove by induction on $n$. When $n=1$, the conclusion holds as we mentioned at the beginning. Now assume that $n>1$ and the theorem is true for $<n$.

Suppose that char $R=p$; then $\delta=d^{p}$ is also a derivation on $R$. If $n$ is divisible by $p$, the assumption reads $\delta^{n / p}(R) \subseteq Z$. Since $n / p<n$, it follows from induction hypothesis that either $d^{n}=\delta^{n / p}=0$ or $R$ is commutative. So we may henceforth assume that $R$ is of characteristic 0 or a prime $p$ not dividing $n$.

For $\alpha \in Z$ and $x \in R$, we have $d^{n}\left(\alpha d^{n-1}(x)\right) \in Z$. That is,

$$
d^{n}(\alpha) d^{n-1}(x)+\sum_{i=1}^{n}\left(\begin{array}{c}
n \\
i
\end{array}\right) d^{n-i}(\alpha) d^{n+i-1}(x) \in Z
$$

for all $\alpha \in Z, x \in R$. Note that each term in the summation is already in $Z$, therefore $d^{n}(Z) d^{n-1}(R) \subseteq Z$. Consequently, either $d^{n}(Z)=0$ or $d^{n-1}(R) \subseteq Z$. If $d^{n-1}(R) \subseteq Z$ we are done by induction hypothesis. So we assume that $d^{n}(Z)=0$.

Received by the editors July 29, 1985 and, in revised form, September 4, 1985. 1980 Mathematics Subject Classification (1985 Revision). Primary 16A72.

Key words and phrases. Prime rings, derivations. 
Let $m$ be the smallest integer such that $d^{m}(Z)=0$. Assume first that $m \geqslant 2$. For $\alpha \in Z$ and $x \in R$ we have

$$
d^{n}\left(d^{m-2}(\alpha) x\right)=d^{m-2}(\alpha) d^{n}(x)+n d^{m-1}(\alpha) d^{n-1}(x) \in Z .
$$

Since $d^{m-2}(\alpha) d^{n}(x) \in Z$, we have $n d^{m-1}(\alpha) d^{n-1}(x) \in Z$ for all $\alpha \in Z, x \in R$. Recall that char $R$ is 0 or $p+n$, so $d^{m-1}(Z) d^{n-1}(R) \subseteq Z$ follows. By the minimality of $m, d^{m-1}(Z) \neq 0$ so $d^{n-1}(R) \subseteq Z$, and we are done. Hence it remains to check the case when $m=1$, that is, $d(Z)=0$.

Since $Z \neq 0$, we may localize $R$ at $Z$ to get a ring $S=\{a / \alpha \mid a \in R, \alpha \in Z, \alpha \neq$ 0 \}. Then $S$ is a prime ring with center the quotient field of $Z$. Moreover, we can extend $d$ to a derivation $\bar{d}$ on $S$ by defining $\bar{d}(a / \alpha)=d(a) / \alpha$. Then $\bar{d}^{n}(S) \subseteq Z(S)$, the center of $S$. So we may replace $R$ and $d$ by $S$ and $\bar{d}$, respectively, and assume that $Z$ is a field. Thus $d^{n}(R)$ is a $Z$-subspace of dimension at most 1 .

Let $k$ be the smallest integer such that $d^{k}(R)$ is finite dimensional over $Z$. Assume that $k \geqslant 1$. For $x, y \in R$ we have

$$
d^{n}\left(d^{k-1}(x) y\right)=d^{k-1}(x) d^{n}(y)+\sum_{i=1}^{n}\left(\begin{array}{c}
n \\
i
\end{array}\right) d^{k+i-1}(x) d^{n-i}(y) \in Z .
$$

Note that $d^{k+i-1}(R)$ is finite dimensional over $Z$, so is $d^{k+i-1}(R) d^{n-i}(y)$ for all $y \in R$ and $i \geqslant 1$. Thus it follows that $d^{k-1}(R) d^{n}(y)$ must be finite dimensional over $Z$ for each $y \in R$. Since $d^{n}(y) \in Z$, we would have $d^{k-1}(R)$ finite dimensional to contradict the choice of $k$ provided $d^{n}(y)$ were not zero. Hence we must have $d^{n}(R)=0$.

At last, assume that $k=0$, that is, $R$ is finite dimensional over $Z$. Being a prime ring, $R$ must be simple. Now $d(Z)=0$, so $d$ must be inner by a classic result [2, Proposition, p. 100]. In other words, there exists $a \in R$ such that $d(x)=a x-x a$ for all $x \in R$. Thus $a d^{n}(x)=d^{n}(a x) \in Z$, and so either $a \in Z$ or $d^{n}(x)=0$ for all $x \in R$. In either case, we have always $d^{n}=0$. This completes the proof.

Next we extend the previous theorem by conditioning $d^{n}(x) \in Z$ merely for all $x$ in some nonzero ideal $I$ of $R$.

THEOREM 2. Let $R$ be a prime ring with center $Z$ and $I$ a nonzero ideal of $R$. Suppose that $d$ is a derivation on $R$ such that $d^{n}(I) \subseteq Z$ for some natural number $n$. Then either $d^{n}=0$ or $R$ is commutative.

Proof. Let $J=I+d(I)+d^{2}(I)+\cdots$. Then $J$ is a nonzero ideal of $R$, $d(J) \subseteq J$, and $d^{n}(J) \subseteq Z$. Applying Theorem 1, we can conclude that either $d^{n}(J)=0$ or $J$ is a commutative ring. However, if $d^{n}(J)=0$, then $d^{n}=0$ by a theorem due to Chung and Luh [1]; while if $J$ is commutative, so is the whole ring $R$. Thus the theorem is proved.

\section{REFERENCES}

1. L. O. Chung and J. Luh, Nilpotency of derivatives on an ideal, Proc. Amer. Math. Soc. 90 (1984), 211-214.

2. I. N. Herstein, Noncommutative rings, Carus Monograph no. 15, MAA, Buffalo, N. Y., 1968.

3. P. H. Lee and T. K. Lee, On derivations of prime rings, Chinese J. Math. 9 (1981), 107-110.

Department of Mathematics, National Taiwan University, Taipei, Taiwan 